\title{
Role of PIR-B in Autoimmune Glomerulonephritis
}

\author{
Toshiyuki Takai, Akira Nakamura, and Shota Endo \\ Department of Experimental Immunology, Institute of Development, Aging and Cancer, Tohoku University, \\ 4-1 Seiryo, Sendai 980-8575, Japan \\ Correspondence should be addressed to Toshiyuki Takai, tostakai@idac.tohoku.ac.jp
}

Received 28 July 2010; Accepted 7 September 2010

Academic Editor: Monica Fedele

Copyright ( $) 2011$ Toshiyuki Takai et al. This is an open access article distributed under the Creative Commons Attribution License, which permits unrestricted use, distribution, and reproduction in any medium, provided the original work is properly cited.

\begin{abstract}
PIR-B, an inhibitory receptor expressed on murine B cells and myeloid cells, regulates humoral and cellular immune responses via its constitutive binding to the ligand, MHC class I molecules, on the same cells (cis) or on different cells (trans). Although it has been speculated that PIR-B is important for maintaining peripheral tolerance, PIR-B single deficiency does not cause overt autoimmune diseases. Recently, however, the combination of its deficiency with the Fas lpr mutation was found to result in augmented production of autoantibodies such as IgG rheumatoid factor and anti-DNA IgG, leading to glomerulonephritis in mice. Although the precise molecular mechanism for the overall scenario is unclear, PIR-B was found to suppress TLR9-mediated production of naturally autoreactive antibodies by innate B cells or B-1 cells by inhibiting the activation of Bruton's tyrosine kinase. Thus, PIR-B is an important regulator of innate immunity mediated by TLR9 in B-1 cells, which can otherwise provoke autoimmunity when overactivated.
\end{abstract}

\section{Introduction}

B cells are continuous producers of natural antibodies with potential reactivities to autologous tissues as well as the on-demand factories of high-affinity antibodies principally against pathogens. Because various self-reactive antibodies are produced in many autoimmune diseases through several elusive mechanisms, a regulatory network for maintaining tolerance to self in B cells while keeping the potential for producing antibodies with specificities and affinities against foreign antigens in sufficient amounts has long been a challenging field for immunologists.

We are now aware of that a series of inhibitory receptors are expressed preferentially on $\mathrm{B}$ cells and other immunoregulatory cells such as dendritic cells (DCs). Thus, the roles of these receptors, including that of the paired immunoglobulin- (Ig-) like receptor (PIR)-B $[1,2]$ in the regulation of $\mathrm{B}$ cells, which we will describe in detail in this paper, have been attracting much interest from many researchers. Some intriguing knowledge on the regulatory role of PIR-B in the immune system has already been reported elsewhere [3-5] (Table 1). Therefore, after briefly overviewing PIR-B's novel characteristics revealed in the past several years, this paper will mainly deal with the recent progress of the understanding of PIR-B-mediated immune regulation, particularly focusing on its role in controlling the production of potential autoantibodies in response to activation via innate-immune stimuli.

\section{PIR-B Is the MHC Class I Receptor in $B$ Cells and Myeloid Cells}

2.1. Recognition of MHC Class I Molecules. In the immune system, there are three types of major histocompatibility complex class I- (MHCI-) recognition molecules. In addition to the well-known $\mathrm{T}$ cell receptor (TCR)-CD8 complex of $\mathrm{CD}^{+} \mathrm{T}$ cells and the killer cell receptors on NK cells, mammalian B cells and myeloid-lineage cells possess the third type of MHCI-recognizing inhibitory receptors, which may constitutively regulate these cells. The latter are murine PIR-B and its close relatives or orthologs in humans, the leukocyte Ig-like receptors (LILR)B1 and LILRB2 [3, 4, 6, 7]. In contrast to the former two types of receptors, which target the polymorphic $\alpha 1 / \alpha 2$ region of MHCI, LILRB molecules were demonstrated to bind a constant $\alpha 3$ domain and $\beta_{2}$ 
microglobulin of MHC $[8,9]$. PIR-B's mode of recognition of MHCI has not been clarified yet, although it is speculated to be essentially the same as that of LILRB [4].

2.2. Protein Structure. PIR-B is a type I transmembrane glycoprotein comprising six extracellular Ig-like domains, a hydrophobic transmembrane segment, and a cytoplasmic portion with four immunoreceptor tyrosine-based inhibitory motif (ITIM) or ITIM-like sequences (Figure 1). Comparison of the available sequences of PIR extracellular portions from 129/Sv, B10.A, and BALB/c mice indicated fairly high sequence similarity, but multiple substitutions of amino acid residues were observed like in LILR molecules [23], especially in the first four ectodomains $[2,24]$. It is not known whether these polymorphisms could modulate the binding of PIR-B to various MHCI and its inhibitory functions.

2.3. Expression. PIR-B is expressed on B cells and myeloidlineage cells including mast cells, macrophages, granulocytes, DCs, and osteoclasts, but not on thymocytes, mature $\mathrm{T}$ cells, or NK cells $[1,2,25,26]$. PIR-B expression takes place mostly in a pairwise fashion with PIR-A, an activating isoform associated with the $\mathrm{Fc}$ receptor common $\gamma$ subunit $(\mathrm{FcR} \gamma)$, as judged on Western blot analysis [25]. In many cases, however, the cell-surface expression of PIR-B occurs more predominantly than that of PIR-A, as judged on flow cytometric analysis of PIR-B- or FcR $\gamma$-deficient cells with a specific monoclonal antibody $(\mathrm{mAb}), 6 \mathrm{C} 1$, that targets a shared epitope of PIR-B and PIR-A [10, 14, 25]. In mature naive $B$ cells, PIR-B is exclusive on the surface [10]. The cell surface levels of PIR molecules on myeloid- and B-lineage cells increase with cellular differentiation and activation [25]. The PIR-B level is highest on marginal zone (MZ) B cells, and B-1 cells express higher PIR-B levels than B-2 cells $[11,25]$.

2.4. Unexpected Role in the Neuronal System. Recently, it was reported that PIR-B and one of its human orthologs, LILRB2, are expressed on neuronal cells $[20,21]$. In addition, Atwal et al. [21] have identified three proteins expressed in the central nervous system as the novel physiological ligands for PIR-B and LILRB1. These are neurite outgrowth inhibitor protein (Nogo)A, myelin-associated glycoprotein (MAG or Siglec-4), and oligodendrocyte myelin glycoprotein (OMgp) [21]. This finding suggests a novel notion that PIR-B is not only a receptor of MHCI for immune regulation but also a determinant of neuronal regeneration responding to the multiple ligands in the central nervous system. These issues have recently been reviewed extensively elsewhere [27, 28].

\section{Signal Regulation by PIR-B}

3.1. Cis/Trans Binding to MHCI. Considering the ubiquitous expression of MHCI molecules on hematopoietic as well as nonhematopoietic cells, the question arises as to whether PIR-B can recognize MHCI on the same cells (in cis), on different cells (in trans), or both. It has been shown that inhibitory Ly49A expressed on murine NK cells not only binds to its $\mathrm{H}-2 \mathrm{D}^{\mathrm{d}}$ ligand expressed on the potential target cells in trans but also is constitutively associated with $\mathrm{H}$ $2 \mathrm{D}^{\mathrm{d}}$ in cis [29]. The cis association and trans interaction occur through the same binding site. Consequently, the cis association restricts the number of Ly49A receptors available for the trans binding of $\mathrm{H}-2 \mathrm{D}^{\mathrm{d}}$ on target cells and reduces NK cell inhibition by lowering the threshold at which NK cell activation exceeds NK cell inhibition [30-32]. This notion, however, has not been verified to be the rule for every inhibitory receptor that binds to its physiological ligand expressed in the own tissues.

Our study on mast cell regulation by PIR-B in the context of allergic responses in Pirb $^{-/}$mice verified that PIR-B on mast cells binds to MHCI molecules in cis, as judged on fluorescence resonance energy transfer (FRET) analysis [14]. In addition, FRET revealed that the cis interaction was also the case for the interaction between MHCI and LILRB2 expressed on human basophilic leukemia KU812 cells. Interestingly, mast cell responses to stimulation by IgE crosslinking or lipopolysaccharides were suppressed to a significant extent by such a cis interaction on the mast cell surface. In both PIR-B and $\beta 2$-microglobulin, its ligand, deficiency, both the in vitro cytokine responses and in vivo anaphylactic responses were significantly augmented, suggesting the physiological importance of the cis interaction [14]. Our analysis of osteoclast precursor cells revealed that the development of osteoclasts is also regulated by PIR-B and that this regulatory mechanism involves the cis interaction of PIR-B-MHCI on osteoclast precursor cells [26]. The cis interaction between PIR-B and MHCI was found also on other cell surfaces such as that of B cells and DCs [13].

PIR-B on DCs and MHCI on $\mathrm{CD}^{+} \mathrm{T}$ cells were found to interact in trans at the immunological synapse. Moreover, $\mathrm{CD}^{+} \mathrm{T}$ cells were found to be more activated upon interaction with $\mathrm{Pirb}^{-/-}$DCs than wild-type cells. This observation was unexpected, because it was known that Pirb $^{-/}$DCs could not support sufficient $\mathrm{CD}^{+} \mathrm{T}$ cell activation toward Th1 development due to their insufficient maturation in PIR-B deficiency and reduced secretion of IL12 [10]. Considering the possibility that MHCI molecules on DCs could play a role as a shared ligand for CD8 on $\mathrm{T}$ cells and for PIR-B on the DCs themselves, it would be interesting to test the hypothesis that CD8 and PIR-B could be competitive as to binding to MHCI on DCs, and it was demonstrated that this was the case [13]. Surface plasmon resonance analysis revealed that PIR-B and CD $8 \alpha \alpha$ compete in binding to MHCI. In vitro and in vivo analyses also verified that irrb $^{-/-}$DC provoked cytotoxic T lymphocytes (CTLs) more efficiently, leading to accelerated rejection of skin grafts and tumors. Thus, PIR-B regulates CTL triggering by blocking the access of CD8 molecules to MHCI. It remains to be determined whether or not PIR-B on DCs is more prone to bind $\mathrm{MHCI}$ in trans on $\mathrm{CD}^{+} \mathrm{T}$ cells than the binding in cis when an immunological synapse is induced between $\mathrm{DC}-\mathrm{CD} 8^{+} \mathrm{T}$ cells.

3.2. Immune Regulation. Studies on Pirb $^{-/-}$mice during the past 10 years have provided us with an insight into 
TABle 1: Phenotypes observed in Pirb $^{-/-}$mice and Pirb transgenic (tg) mice.

\begin{tabular}{|c|c|c|c|}
\hline Mice & Cells $^{\mathrm{a}}$ & Phenotypes $^{\mathrm{b}}$ & References \\
\hline \multirow{13}{*}{$\operatorname{Pirb}^{-/-}$} & B-2 cells & $\begin{array}{l}\text { Enhanced proliferation upon BCR } \\
\text { stimulation }\end{array}$ & {$[10]$} \\
\hline & B-1 cells & $\begin{array}{l}\text { Enhanced proliferation and autoantibody } \\
\text { production upon } \mathrm{CpG} \text { stimulation }\end{array}$ & {$[11]$} \\
\hline & \multirow[t]{2}{*}{ DCs } & Impaired maturation & {$[10]$} \\
\hline & & Exacerbated graft-versus-host disease & {$[12]$} \\
\hline & $\mathrm{CD}^{+} \mathrm{T}$ cells & Augmented activation & {$[13]$} \\
\hline & Mast cells & Augmented anaphylaxis & {$[14]$} \\
\hline & Macrophages & $\begin{array}{l}\text { Augmented cytokine and chemokine } \\
\text { signaling }\end{array}$ & {$[15]$} \\
\hline & Neutrophils & Augmented integrin signaling & {$[16]$} \\
\hline & Eosinophils & Enhanced recruitment in Th2 response & {$[17]$} \\
\hline & Macrophages $^{c}$ & Sensitive to Salmonella infection & {$[18]$} \\
\hline & Macrophages & Reduced binding of Staphylococcus aureus & {$[19]$} \\
\hline & \multirow[t]{2}{*}{ Neuronal cells } & $\begin{array}{l}\text { Enhanced plasticity of visual nerve } \\
\text { connections }\end{array}$ & {$[20]$} \\
\hline & & Enhanced neurite outgrowth & {$[21]$} \\
\hline \multirow{2}{*}{ Pirb tg } & Thymocytes & Normal development & {$[22]$} \\
\hline & Mature T cells & Impaired Th1 response & {$[22]$} \\
\hline
\end{tabular}

${ }^{a}$ Cells involved mainly in the observed phenotype.

${ }^{\mathrm{b}}$ Major phenotypes observed.

${ }^{\mathrm{c}}$ Unidentified, though involving macrophages.

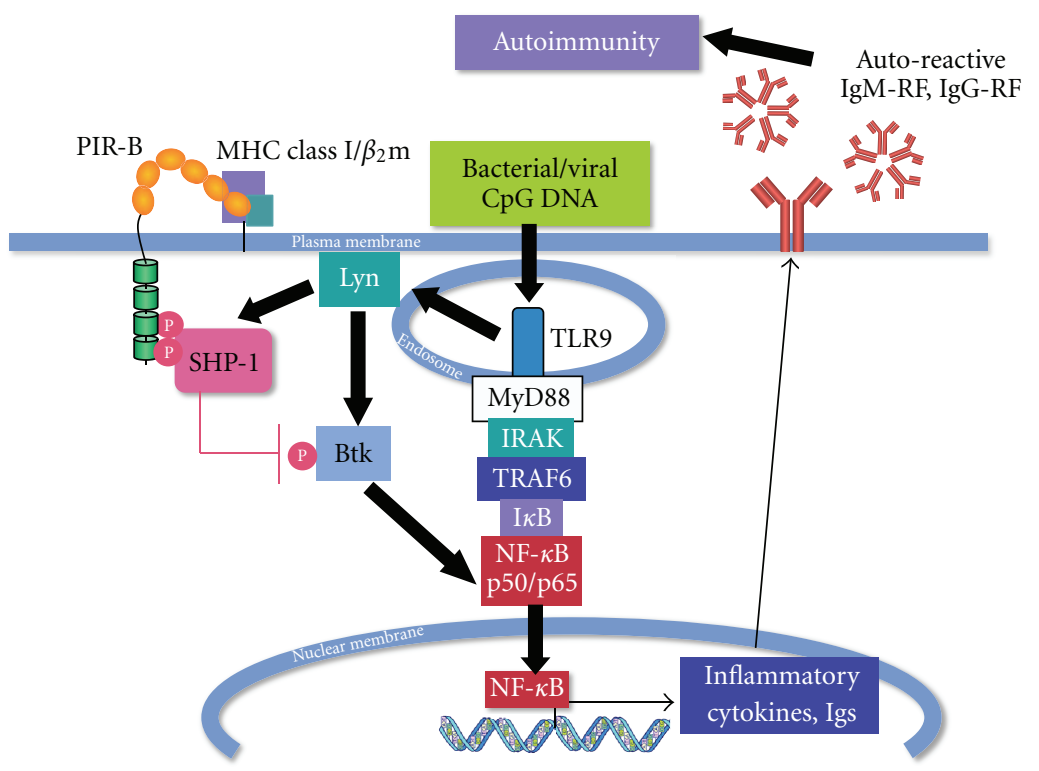

FIgure 1: Proposed mechanism for PIR-B-mediated regulation of TLR9-induced autoantibody production in B-1 cells. Unmethylated CpG-harboring DNA of bacterial and viral origin stimulates TLR9 in the endosomal compartments of various cells including B-1 cells via signal relay from MyD88, IRAK, and TRAF6, and then I $\kappa$ B phosphorylation, nuclear translocation of NF- $\kappa \mathrm{B}$, and initiation of the transcription of various mRNAs of innate responses, including those for autoreactive natural antibodies. Excess activation of the TLR9 system is thus potentially harmful, because excessive production of autoreactive IgM and, particularly, that switched to IgG might cause autoimmune disease. TLR9 in B-1 cells is regulated by PIR-B via Btk intersection, in which the phosphorylation of PIR-B is augmented immediately through TLR9-initiated Lyn activation, and the concomitantly enhanced SHP-1 recruitment to the phospho-ITIMs of PIR-B leads to accelerated dephosphorylation of Btk, which then attenuates the phosphorylation level of NF- $\kappa \mathrm{B}$ p65RelA. Constitutive association of PIR-B with MHCI in cis on the surface of B-1 cells may be crucial for maintaining the TLR9 cascade being not overactivated, and once activated by CpG, immediate early suppression will occur via augmented SHP-1 recruitment to PIR-B-MHCI. 
the physiological significance of the MHCI recognition by PIR-B in the immune responses, especially in $\mathrm{B}$ cell activation, antigen presentation, humoral immunity, and transplantation (Table 1), most of which have already been described elsewhere [3-5]. This section deals with the recent findings on the PIR-B's functions in bacterial infection, eosinophil signaling, and T cell functions briefly, and in the next section we will focus on its roles in autoimmunity.

3.2.1. Eosinophil Function. Munitz et al. [17] described a positive and negative dual regulatory role of PIR-B in Th2 inflammation, in which PIR-B shows overexpression on eosinophils. Based on the different responses to eotaxin- or leukotriene $\mathrm{B}_{4}$-dependent chemotaxis of Pirb ${ }^{-/-}$eosinophils, it was found that extracellular signal-related kinase 1 and 2 or Erk1/2 phosphorylation in response to these stimulators was opposite in eosinophils in vitro. It was proposed that an inhibitory receptor can exhibit dual functionality in distinct cell types after unique cellular signals [17]. This interesting hypothesis will have to be evaluated in other cellular and experimental settings as well as in other receptor systems, which might shift our idea of merely "inhibitory" receptors to more sophisticated "regulatory" ones.

3.2.2. T Cell Development and Function. PIR-B is expressed on immature and mature B cells, and myeloid cells but not on NK cells or T cells. However, besides the speculation that PIR-B should be under the control of $\mathrm{B} /$ myeloid-specific transcription factors, it has been unknown why $\mathrm{T}$ cells and NK cells preferentially use inhibitory coreceptors such as CTLA-4, PD-1, and KIRs, but not PIR-B. This issue should be considered how PIR-B expression is regulated during prethymic progenitor cells, thymocytes, and mature $\mathrm{T}$ cells, referring functional consequences when PIR-B is genetically deleted or expressed by force.

Masuda et al. [33] serendipitously found that PIR immunoreactivity was transiently detected on very early prethymic progenitors of T/NK/DCs, although PIR is not found on thymocytes or mature $\mathrm{T}$ cells on flow cytometry and immunohistochemistry with $\mathrm{mAb} 6 \mathrm{C} 1$ recognizing both PIR-A and PIR-B [25]. PIR expression was maintained until the earliest intrathymic stage and then downregulated before the onset of CD25 expression, which is a hallmark of the earliest stage of double negative thymocytes [33], suggesting that PIR-B expression should be down modulated during thymic development. Then, for what is it suppressed? The above findings prompted us firstly to define in more detail the cells at prethymic and intrathymic stages of $\mathrm{T}$ cell development in PIR-B deficiency. We found that PIR-B, not PIR-A, is the receptor expressed in the prethymic stage of T-lineage cells. We then examined the hypothesis that PIR$\mathrm{B}$ is not only a regulator of early $\mathrm{T}$ cell development but is also detrimental if expressed on mature $\mathrm{T}$ cells [22]. Unexpectedly, analysis of the possible influence of PIR-B revealed no abnormalities in the development of immature and mature $\mathrm{T}$ cells when PIR-B was expressed ectopically at intrathymic stages or on peripheral $\mathrm{T}$ cells. Also, genetic depletion of PIR-B did not cause any obvious alterations in prethymic $\mathrm{T}$ progenitors. Interestingly, upon antigenic or allogeneic stimulation, however, peripheral $\mathrm{T}$ cells with ectopic PIR-B showed a reduced Th1 response, due to suppression of proximal TCR signaling by constitutive cis binding of PIR-B to MHCI on the same cell surface. These results suggest that $\mathrm{T}$ cell expression of PIR-B is strictly prohibited at the periphery so as to secure prompt immune responses in urgent situations such as infections by fastmultiplying bacteria. Although it remains to be determined why prethymic early T/NK/DC progenitors express PIR-B, it is possible that mature $\mathrm{T}$ cells select a transient inhibitory system like CTLA-4, whose expression is induced upon activation, instead of a constitutive inhibitory system such as PIR-B.

3.2.3. Bacterial Infection. The influence of PIR-B deficiency was examined in infections with bacteria such as Staphylococcus aureus and Salmonella enterica $[18,19]$. Pirb $^{-/-}$mice were more susceptible to Salmonella infection than wild-type C57BL/6 mice [18]. The susceptibility was in part due to the fact that $\mathrm{Pirb}^{-/-}$macrophages failed to control intracellular replication of Salmonella in vitro, possibly due to imbalance between PIR-A and PIR-B in the protective immune response to bacteria [18]. Nakayama et al. [19] found that PIR$\mathrm{B}$, and human LILRB1 and LILRB3 act as cell surface receptors for S. aureus and Escherichia coli among various pattern recognition receptors including Toll-like receptors (TLRs), scavenger receptors, and lectins expressed on the innate immune cell surface. In mouse bone marrow-derived macrophages, PIR-B masking by $\mathrm{mAb}$ or genetic deletion of PIR-B caused an enhanced TLR-mediated inflammatory response to bacteria [19]. It remains unclear whether such inhibition of TLR signaling by PIR-B is beneficial or harmful as to innate and adaptive responses to bacterial infections in vivo. Nonetheless, the observations in this study suggest that PIR-B plays multifaceted roles by recognizing multiple ligands, including bacterial cell surface component(s) in addition to MHCI and neuronal ligands. In line with these observations, Munitz et al. [34] found very recently that PIR$B$ has an inhibitory role in macrophage activation during intestinal inflammatory responses, such as dextran sodium sulfate-induced colitis, and in response to E. coli.

\section{TLR9 Regulation in B-1 Cells and Autoimmunity}

4.1. B-1 Cells and Natural Antibodies. Setting aside the reason for their expression, many inhibitory receptors are preferentially expressed on B cells. These include the IgG Fc receptor Fc $\gamma$ RIIB $[35,36]$, a carbohydrate receptor, CD22 $[37,38]$, the CD100 receptor CD72 [39], a sialoglycoprotein receptor, Siglec-G [40], a carbohydrate receptor, CD45 [41], and the MHCI receptor PIR-B. CD45 is a phosphatase itself, and all other receptors described above recruit $\mathrm{SH} 2$ domaincontaining tyrosine phosphatase (SHP)-1 and/or SHP-2, or inositol phosphatase (SHIP) upon stimulation [40].

In mice, there are four subsets of B cells. Two subsets of mature naive B cells termed follicular B cells or 
conventional B cells $\left(\mathrm{B} 220^{+} \mathrm{CD} 23^{\text {high }} \mathrm{CD} 21^{\text {low }}\right)$ and marginal zone (MZ) B cells $\left(\mathrm{B} 220^{+} \mathrm{CD} 23^{\text {low }} \mathrm{CD} 21^{\text {high }}\right)$ are located primarily in secondary lymphoid organs such as the spleen [42]. Follicular B cells participate in the responses to $\mathrm{T}$ cell-dependent antigens and in the production of highaffinity IgG antibodies, while MZB cells participate in the response to blood-borne bacterial antigens. On the other hand, two other subsets of mature naive B cell populations reside mainly in the peritoneal and pleural cavities. These include B-1a (B220 low $\left.\mathrm{IgM}^{\text {high }} \mathrm{CD} 11 \mathrm{~b}^{+} \mathrm{CD}^{+}\right)$ and $\mathrm{B}-1 \mathrm{~b}\left(\mathrm{~B} 220^{\text {low }} \mathrm{IgM}^{\text {high }} \mathrm{CD} 11 \mathrm{~b}^{+} \mathrm{CD}^{-}\right)$cells. Therefore, conventional B cells are often called B-2 cells. Although the precise roles of these B-1 cell populations are still not well understood, B-1b cells are mostly responsible for the adaptive immune responses to $\mathrm{T}$-independent antigens and exert a memory function, while B-1a cells spontaneously produce natural IgM antibodies with broad specificities, thereby contributing to innate immunity $[43,44]$.

It has been suggested that innate $\mathrm{B}$ cells, including MZ B, B-1a, and B-1b ones, can be potentially harmful for maintaining peripheral tolerance, because B-1a cells, in particular, continuously produce large amounts of IgM natural antibodies weakly reactive to autologous tissues, but this remains controversial. The natural IgM antibodies can be switched to IgG, without BCR stimulation in response to pathogen-associated molecular patterns (PAMPs) such as bacterial cell wall components, and hypomethylated $\mathrm{CpG}$ motif-containing DNA of bacterial and viral origin $[42,45]$. Some PAMPs are recognized by TLRs expressed on the cell surface or in endosomal compartments. Murine B cells of all subsets express several TLRs [42]. Therefore, excess stimulation of the TLR pathway in B cells such as in B1a ones is supposed to be connected with provocation of autoimmunity. In fact, B-1 cells express a set of TLRs, including TLR4, TLR7, and TLR9 [42, 46]. Several lines of evidence indicate the importance of the regulation of TLR signaling in B-1 cells, which prevents over-stimulation of TLRs so as not to evoke overproduction of natural antibodies including potentially harmful autoantibodies $[46,47]$. Such regulatory mechanisms for TLRs in B-1 cells may include inhibitory receptors, and we speculated that PIR-B could play a role here.

Interestingly, in $\mathrm{Pirb}^{-/-}$mice the peritoneal B-1a cell population significantly increases, particularly with aging, compared to in wild-type C57BL/6 mice [10]. However, it has not been determined how the B-1a cell compartment is regulated by PIR-B or what the physiological or pathological consequence of the expanded B-1a cells is in the contexts of infection and autoimmunity. We found that PIR-B on B-1 cells suppresses TLR9 signaling via Bruton's tyrosine kinase (Btk) dephosphorylation, which leads to attenuated activation of NF- $\kappa \mathrm{B}$ p65RelA and blocks the production of natural IgM antibodies, including anti-IgG Fc autoantibodies or rheumatoid factor (RF) in particular [11].

4.2. Autoimmune Glomerulonephritis. Initially we observed that Pirb $^{-/-}$mice were grossly normal and survived well, at least to 50 weeks of age, without any abnormalities in the histology of their tissues including glomeruli. However, $\mathrm{Pirb}^{-/-}$mice with the $l p r$ mutation of Fas ( Pirb $^{-/-} \mathrm{Fas}^{l \mathrm{l} r}$ mice) were markedly short lived, because of a deficit in renal function due to the development of glomerulonephritis with the depositing of $\operatorname{IgG}$, IgM, and C3. While the levels of IgM- and IgG-RF were high in Fas ${ }^{l p r}$ mice, these levels were further elevated in $\mathrm{Pirb}^{-/-} \mathrm{Fas}^{\mathrm{lpr}}$ animals. On the other hand, the serum levels of anti-dsDNA and -ssDNA autoantibodies were also high in Fas ${ }^{l p r}$ mice, but they were not elevated further in $\mathrm{Pirb}^{-/-} \mathrm{Fas}^{\mathrm{lPr}}$ mice, indicating that PIR-B deficiency has a marked impact on the augmentation of RF production in vivo. The peritoneal B-1a cell and splenic plasma cell populations were also increased in $\mathrm{Pirb}^{-/-} \mathrm{Fas}^{\mathrm{lpr}}$ mice. From these data, it was concluded that PIR-B deficiency in combination with $\mathrm{Fas}^{l p r}$ renders mice susceptible to the development of autoimmune glomerulonephritis, which is accompanied by significantly elevated RF production with robust production of other autoantibodies including those against self DNA.

4.3. TLR9 Signal in B-1 Cells. RF is one of the autoreactive natural antibodies reacting with the $\mathrm{Fc}$ portion of $\operatorname{IgG}$, whose primary role is believed to be as the first line defense against infections. It is also a marker of rheumatoid arthritis and other connective tissue diseases such as systemic lupus erythematosus and infectious diseases. However, the pathogenicity of RF in autoimmune diseases has not been established sufficiently. Like other natural autoreactive antibodies such as those reactive with phosphatidylcholine, the main source of RF is considered to be B-1 cells [48]. To determine the precise source, the RF levels in sera from B6 and $\mathrm{Pirb}^{-/-}$mice were examined in the absence of $\mathrm{Fas}^{\mathrm{l}}{ }^{\mathrm{pr}}$, which might have a great influence on the RF level.

Given the augmented RF production and enlarged B1a cell population in $\mathrm{Pirb}^{-/-} \mathrm{Fas}^{\mathrm{lpr}}$ mice, our attention next turned to the characteristics of Pirb ${ }^{-1-}$ B-1 cells. Pirb ${ }^{-/-}$B1 cells proliferated particularly well upon stimulation with CpG (TLR9 ligand) compared to wild-type ones, indicating an important role of TLR9 in B-1 cell activation and its regulation by PIR-B. Total IgM, IgM-RF, and IL-10 release was also more elevated in Pirb $^{-1}$ B-1 cells than in wild-type ones. While B-1a and B-1b cells express PIR-B molecules more abundantly on their surface than B-2 cells [25], the expression of PIR-A, an activating counterpart of PIR-B, was not detected on B-1a cells [11], suggesting the presence of a PIR-B-mediated inhibitory signaling that specifically regulates TLR9.

Like in mast cells and splenic B cells stimulated by $\operatorname{IgE}$ receptor or BCR crosslinking, respectively, $\mathrm{CpG}$ induced augmented PIR-B phosphorylation in B-1 cells, which was accompanied by an increase in SHP- 1 recruitment, whereas such events were not observed in TLR9-deficient cells, which led the conclusion that CpG-mediated PIR-B phosphorylation occurs downstream of TLR9 [11]. Preceding studies indicated that, in splenic B cells, Lyn is the major Src family kinase that phosphorylates PIR-B [49]. However, it is not known whether or not this is also the case in peritoneal B-1 cells. The phosphorylation level of Lyn was 
indeed augmented as early as $5 \mathrm{~min}$ after $\mathrm{CpG}$ stimulation of B-1 cells regardless of the presence or absence of PIRB. However, augmented phosphorylation of Lyn was not observed in TLR9-deficient B-1 cells. Also, Lyn and PIR$\mathrm{B}$ were co-immunoprecipitated from a macrophage lysate after stimulation with CpG. These results indicate that Lyn is rapidly activated downstream of TLR9 and strongly suggest that Lyn is a candidate, if not the sole, Src family kinase that phosphorylates PIR-B.

What are the downstream signal cascades, such as the NF- $\kappa$ B and MAP kinase pathways, of TLR9 modulated by PIR-B in B-1 cells? MAPK and Erk were significantly augmented after CpG stimulation, but their levels and kinetics were comparable in normal and $\mathrm{Pirb}^{-/-}$B-1 cells. On the other hand, p65 NF- $\kappa$ B phosphorylation was found to be markedly augmented in $\mathrm{Pirb}^{-/-} \mathrm{B}-1$ cells after $\mathrm{CpG}$ stimulation, indicating that the NF- $\kappa \mathrm{B}$ pathway could be the target step located most downstream of regulation through Lyn-mediated PIR-B phosphorylation (Figure 1).

Then, which molecule mediates the PIR-B-SHP-1initiated inhibitory signaling pathway to the TLR9-NF- $\kappa$ B cascade? In a B cell line, IIA1.6 cells, Syk and Btk were found to be the substrates for SHP-1 recruited to PIR-B ITIMs upon stimulation of BCR [50]. Examination of the possible involvement of these two molecules in the TLR9 pathway revealed that the phosphorylation status of Syk upon $\mathrm{CpG}$ addition to B-1 cells did not show enhancement of the phosphorylation, while Btk phosphorylation was markedly augmented after $\mathrm{CpG}$ addition in both wild-type and $\mathrm{Pirb}^{-/-}$ B-1 cells, and the augmentation was higher in $\mathrm{Pirb}^{-/-}$B1 cells, indicating that Btk is a major substrate for SHP-1 (Figure 1). These results indicate that Btk phosphorylation is dependent, at least partly, on a Src family kinase, most likely Lyn, and that NF- $\kappa$ B p65RelA phosphorylation is also a critical event downstream of Src family kinase activation. The molecular mechanism for the TLR9-initiated Lyn activation is currently not known. Although SHP-1 is a critical regulator of type I interferon production by macrophages and DCs through inhibition of IRAK1 activation downstream of TLR4 [51], augmented IRAK1 activation was not observed in CpGB stimulated Pirb ${ }^{-/-}$B-1 cells [11], suggesting that B-1 cells preferentially utilize Btk as a key intersecting molecule for the inhibitory circuit (Figure 1).

Recent studies have shown that Btk is an important mediator of the TLR9 cascade in addition to MyD88 and TRAF $[52,53]$. Btk is required for NF- $\kappa \mathrm{B}$ activation, participating in the pathway leading to increased phosphorylation of p65RelA activated by TLR8 and TLR9 [53, 54]. As is well known, Btk is a critical kinase for the development and function of B cells including B-1 cells by ensuring BCR signaling, as has been demonstrated in immunocompromised Xid mice and human X-linked agammaglobulinemia, in which Btk is dysfunctional $[55,56]$. Btk deletion in mice induced defective development of B cells including B-1 ones and their function [57]. The finding that Btk is a critical link between the TLR9 cascade and the PIR-B-mediated regulatory loop, particularly in B-1 cells expressing abundant PIR-B [11], provides an insight into the mechanism underlying the crosstalk between the innate immune system and ITIMharboring receptors.

4.4. Autoimmunity and PIR-B: Questions Remaining. How are the observations on TLR9 signaling in B-1 cells relevant to the prevention of autoimmunity? In this context, several feedback regulators for TLR9 activation have been identified. These include SOCS1 [58], ATF3 [59], IRF-4 [60], and SHP1 [51]. For example, SOCS1-mediated feedback regulation seems to occur after several hours of TLR9 activation followed by inflammatory cytokine induction [58]. It is very characteristic that the PIR-B-mediated suppression of the TLR9 cascade takes place at several minutes after $\mathrm{CpG}$ addition to B-1 cells. Therefore, PIR-B in the TLR9 of B-1 cells does not seem to be a feedback regulatory element but an immediate-early regulator just like the one in mast cell regulation after IgE receptor crosslinking $[14,61]$ and in B-2 cells upon BCR stimulation $[10,62,63]$.

Can RF produced by B-1 cells trigger or exacerbate glomerulonephritis in $\mathrm{Pirb}^{-/-} \mathrm{Fas}^{\mathrm{lpr}}$ mice? $\mathrm{CD}^{+} \mathrm{B}-1$ cells or $\mathrm{B}-1 \mathrm{a}$ cells are considered to be involved in some autoimmune diseases in which RF is frequently detected or RF production is directly coupled to the disease [64-66]. Also, $\mathrm{RF}^{+} \mathrm{B}$ cells are effectively activated via the IgG2a-chromatin immune complex and synergistic stimulation of TLR9-mediated signaling [67]. It is additionally known that RF can bind to anti-dsDNA autoantibodies and form immune complexes [68]. Also, it has been reported that RF itself exhibits cryoglobulin activity and causes glomerulonephritis without any other additional factors [69]. Therefore, glomerulonephritis in Pirb $^{-/-}$Fas $^{l p r}$ mice exhibiting markedly and specifically elevated IgM- and IgG-RF production suggests that these anti-IgG Fc autoantibodies worsen the disease, presumably by accelerating the deposition of IgG autoantibodies against glomerular basement membranes.

Do other TLRs, such as TLR4 and TLR7, in B-1 and B-2 cells possess a similar regulatory system involving PIR$\mathrm{B}$ to that of TLR9? In this regard, it is of note that TLR4mediated cytokine production by mast cells is also negatively regulated by PIR-B [14], although the mechanism has yet to be elucidated. Therefore, it is likely that some TLR-mediated activation cascades in B cells could be linked to the PIR-BSHP-1-mediated suppression in addition to the case of TLR9. On the other hand, TLR4 response in macrophages was not affected by PIR-B $[19,34]$. The reason why TLR4-induced responses are regulated differentially in the differential cell populations is not known currently. However, one may speculate that different cells may be differently dependent on any TLR4-initiated, PIR-B-mediated regulatory pathway such as the Lyn-PIR-B-SHP-1-Btk axis identified in B-1 cells [11]. Such regulation of an innate immune response via TLRs by constitutive and activation-dependent immediateearly suppression of PIR-B could be promising for preventing autoimmunity.

\section{Concluding Remarks}

In recent studies on $\mathrm{Pirb}^{-/-}$mice, there were two important observations. Firstly, the TLR9 system in B-1 cells is 
regulated by PIR-B via the Btk intersection, at which PIRB phosphorylation is immediately augmented by TLR9initiated Lyn activation, and the concomitant enhancement of SHP-1 recruitment to augmented phospho-ITIMs of PIR$B$ leads to Btk dephosphorylation, which then attenuates the phosphorylation level of NF- $\kappa$ B p65RelA. Secondly, excessive production of RF together with other autoantibodies by B-1 cells can be linked to glomerulonephritis with IgG immune complex deposition. Considering these observations, it is becoming increasingly important for manipulation of the PIR-B-mediated inhibitory system in the regulation of autoimmune disease while maintaining the integrity of TLR9-mediated microbial sensing, in such cases as rheumatoid arthritis. Understanding of the PIR-B system in B-1 cells may lead to the development of novel and effective ways of controlling autoimmune diseases.

\section{Acknowledgments}

The authors thank Nicholas Halewood for the editorial assistance, and colleagues in their laboratory for critical reading of the paper. This paper was supported by the Core Research for Evolutional Science and Technology Program of the Japan Science and Technology Agency, a Grant-inAid from the Ministry of Education, Culture, Sports, Science and Technology of Japan, and a grant from the Global COE Program for Network Medicine.

\section{References}

[1] K. Hayami, D. Fukuta, Y. Nishikawa et al., "Molecular cloning of a novel murine cell-surface glycoprotein homologous to killer cell inhibitory receptors," Journal of Biological Chemistry, vol. 272, no. 11, pp. 7320-7327, 1997.

[2] H. Kubagawa, P. D. Burrows, and M. D. Cooper, "A novel pair of immunoglobulin-like receptors expressed by B cells and myeloid cells," Proceedings of the National Academy of Sciences of the United States of America, vol. 94, no. 10, pp. 5261-5266, 1997.

[3] T. Takai, "Paired immunoglobulin-like receptors and their MHC class I recognition," Immunology, vol. 115, no. 4, pp. 433-440, 2005.

[4] T. Takai, "A novel recognition system for MHC class I molecules constituted by PIR," Advances in Immunology, vol. 88, pp. 161-192, 2005.

[5] H. Kubagawa, M. D. Cooper, C. C. Chen et al., "Paired immunoglobulin-like receptors of activating and inhibitory types," Current Topics in Microbiology and Immunology, vol. 244, pp. 137-149, 1999.

[6] M. Colonna, H. Nakajima, and M. Cella, "A family of inhibitory and activating Ig-like receptors that modulate function of lymphoid and myeloid cells," Seminars in Immunology, vol. 12, no. 2, pp. 121-127, 2000.

[7] M. Colonna, "Inhibitory receptors: friend or foe?" Lancet, vol. 361, no. 9363, pp. 1067-1068, 2003.

[8] M. Shiroishi, K. Kuroki, L. Rasubala et al., "Structural basis for recognition of the nonclassical MHC molecule HLA-G by the leukocyte Ig-like receptor B2 (LILRB2/LIR2/ILT4/CD85d)," Proceedings of the National Academy of Sciences of the United States of America, vol. 103, no. 44, pp. 16412-16417, 2006.
[9] B. E. Willcox, L. M. Thomas, and P. J. Bjorkman, "Crystal structure of HLA-A2 bound to LIR-1, a host and viral major histocompatibility complex receptor," Nature Immunology, vol. 4, no. 9, pp. 913-919, 2003.

[10] A. Ujike, K. Takeda, A. Nakamura, S. Ebihara, K. Akiyama, and T. Takai, "Impaired dendritic cell maturation and increased $\mathrm{T}_{\mathrm{H}} 2$ responses in PIR-B ${ }^{-/-}$mice," Nature Immunology, vol. 3, no. 6, pp. 542-548, 2002.

[11] T. Kubo, Y. Uchida, Y. Watanabe et al., "Augmented TLR9induced Btk activation in PIR-B-deficient B-1 cells provokes excessive autoantibody production and autoimmunity," Journal of Experimental Medicine, vol. 206, no. 9, pp. 1971-1982, 2009.

[12] A. Nakamura, E. Kobayashi, and T. Takai, "Exacerbated graft-

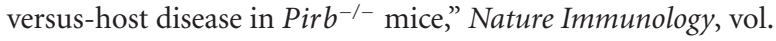
5, no. 6, pp. 623-629, 2004.

[13] S. Endo, Y. Sakamoto, E. Kobayashi, A. Nakamura, and T. Takai, "Regulation of cytotoxic T lymphocyte triggering by PIR-B on dendritic cells," Proceedings of the National Academy of Sciences of the United States of America, vol. 105, no. 38, pp. 14515-14520, 2008.

[14] A. Masuda, A. Nakamura, T. Maeda, Y. Sakamoto, and T. Takai, "Cis binding between inhibitory receptors and MHC class I can regulate mast cell activation," Journal of Experimental Medicine, vol. 204, no. 4, pp. 907-920, 2007.

[15] S. Pereira, H. Zhang, T. Takai, and C. A. Lowell, "The inhibitory receptor PIR-B negatively regulates neutrophil and macrophage integrin signaling," Journal of Immunology, vol. 173, no. 9, pp. 5757-5765, 2004.

[16] H. Zhang, F. Meng, C.-L. Chu, T. Takai, and C. A. Lowell, “The Src family kinases Hck and Fgr negatively regulate neutrophil and dendritic cell chemokine signaling via PIR-B," Immunity, vol. 22, no. 2, pp. 235-246, 2005.

[17] A. Munitz, M. L. McBride, J. S. Bernstein, and M. E. Rothenberg, "A dual activation and inhibition role for the paired immunoglobulin-like receptor B in eosinophils," Blood, vol. 111, no. 12, pp. 5694-5703, 2008.

[18] I. Torii, S. Oka, M. Hotomi et al., "PIR-B-deficient mice are susceptible to Salmonella infection," Journal of Immunology, vol. 181, no. 6, pp. 4229-4239, 2008.

[19] M. Nakayama, D. M. Underhill, T. W. Petersen et al., "Paired Ig-like receptors bind to bacteria and shape TLR-mediated cytokine production," Journal of Immunology, vol. 178, no. 7, pp. 4250-4259, 2007.

[20] J. Syken, T. GrandPre, P. O. Kanold, and C. J. Shatz, "PirB restricts ocular-dominance plasticity in visual cortex," Science, vol. 313, no. 5794, pp. 1795-1800, 2006.

[21] J. K. Atwal, J. Pinkston-Gosse, J. Syken et al., "PirB is a functional receptor for myelin inhibitors of axonal regeneration," Science, vol. 322, no. 5903, pp. 967-970, 2008.

[22] M. Imada, K. Masuda, R. Satoh et al., "Ectopically expressed PIR-B on T cells constitutively binds to MHC class I and attenuates T helper type 1 responses," International Immunology, vol. 21, no. 10, pp. 1151-1161, 2009.

[23] M. Colonna, F. Navarro, T. Bellón et al., "A common inhibitory receptor for major histocompatibility complex class I molecules on human lymphoid and myelomonocytic cells," Journal of Experimental Medicine, vol. 186, no. 11, pp. 18091818, 1997.

[24] Y. Yamashita, D. Fukuta, A. Tsuji et al., "Genomic structures and chromosomal location of p91, a novel murine regulatory receptor family," Journal of Biochemistry, vol. 123, no. 2, pp. 358-368, 1998. 
[25] H. Kubagawa, C.-C. Chen, L. H. Ho et al., "Biochemical nature and cellular distribution of the paired immunoglobulinlike receptors, PIR-A and PIR-B," Journal of Experimental Medicine, vol. 189, no. 2, pp. 309-317, 1999.

[26] Y. Mori, S. Tsuji, M. Inui et al., "Inhibitory immunoglobulinlike receptors LILRB and PIR-B negatively regulate osteoclast development," Journal of Immunology, vol. 181, no. 7, pp. 4742-4751, 2008.

[27] L. M. Boulanger, "Immune proteins in brain development and synaptic plasticity," Neuron, vol. 64, no. 1, pp. 93-109, 2009.

[28] M. T. Filbin, "PirB, a second receptor for the myelin inhibitors of axonal regeneration Nogo66, MAG, and OMgp: implications for regeneration in vivo," Neuron, vol. 60, no. 5, pp. 740742, 2008.

[29] M.-A. Doucey, L. Scarpellino, J. Zimmer et al., "Cis association of Ly49A with MHC class I restricts natural killer cell inhibition," Nature Immunology, vol. 5, no. 3, pp. 328-336, 2004.

[30] L. Scarpellino, F. Oeschger, P. Guillaume et al., "Interactions of Ly49 family receptors with MHC class I ligands in trans and cis," Journal of Immunology, vol. 178, no. 3, pp. 1277-1284, 2007.

[31] J. Back, E. L. Malchiodi, S. Cho et al., "Distinct conformations of Ly49 natural killer cell receptors mediate MHC class I recognition in trans and cis," Immunity, vol. 31, no. 4, pp. 598$608,2009$.

[32] K. E. Andersson, G. S. Williams, D. M. Davis, and P. Höglund, "Quantifying the reduction in accessibility of the inhibitory NK cell receptor Ly49A caused by binding MHC class I proteins in cis," European Journal of Immunology, vol. 37, no. 2, pp. 516-527, 2007.

[33] K. Masuda, H. Kubagawa, T. Ikawa et al., "Prethymic T-cell development defined by the expression of paired immunoglobulin-like receptors," EMBO Journal, vol. 24, no. 23, pp. 4052-4060, 2005.

[34] A. Munitz, E. T. Cole, A. Beichler et al., "Paired immunoglobulin-like receptor B (PIR-B) negatively regulates macrophage activation in experimental colitis," Gastroenterology, vol. 139, no. 2, pp. 530-541, 2010.

[35] S. Bolland and J. V. Ravetch, "Spontaneous autoimmune disease in Fc $\gamma$ RIIB-deficient mice results from strain-specific epistasis," Immunity, vol. 13, no. 2, pp. 277-285, 2000.

[36] T. Takai, "Roles of Fc receptors in autoimmunity," Nature Reviews Immunology, vol. 2, no. 8, pp. 580-592, 2002.

[37] J. G. Cyster and C. C. Goodnow, "Tuning antigen receptor signaling by CD22: integrating cues from antigens and the microenvironment," Immunity, vol. 6, no. 5, pp. 509-517, 1997.

[38] L. Nitschke, R. Carsetti, B. Ocker, G. Köhler, and M. C. Lamers, "CD22 is a negative regulator of B-cell receptor signalling," Current Biology, vol. 7, no. 2, pp. 133-143, 1997.

[39] J. R. Parnes and C. Pan, "CD72, a negative regulator of B-cell responsiveness," Immunological Reviews, vol. 176, pp. 75-85, 2000.

[40] L. Nitschke, "CD22 and Siglec-G: B-cell inhibitory receptors with distinct functions," Immunological Reviews, vol. 230, no. 1, pp. 128-143, 2009.

[41] M. L. Hermiston, J. Zikherman, and J. W. Zhu, "CD45, CD148, and Lyp/Pep: critical phosphatases regulating Src family kinase signaling networks in immune cells," Immunological Reviews, vol. 228, no. 1, pp. 288-311, 2009.

[42] L. Genestier, M. Taillardet, P. Mondiere, H. Gheit, C. Bella, and T. Defrance, "TLR agonists selectively promote terminal plasma cell differentiation of $\mathrm{B}$ cell subsets specialized in thymus-independent responses," Journal of Immunology, vol. 178, no. 12, pp. 7779-7786, 2007.

[43] K. R. Alugupalli, J. M. Leong, R. T. Woodland, M. Muramatsu, T. Honjo, and R. M. Gerstein, "B1b lymphocytes confer T cellindependent long-lasting immunity," Immunity, vol. 21, no. 3, pp. 379-390, 2004.

[44] K. M. Haas, J. C. Poe, D. A. Steeber, and T. F. Tedder, "B-1a and $\mathrm{B}-1 \mathrm{~b}$ cells exhibit distinct developmental requirements and have unique functional roles in innate and adaptive immunity to S. pneumoniae," Immunity, vol. 23, no. 1, pp. 7-18, 2005.

[45] B. He, X. Qiao, and A. Cerutti, "CpG DNA induces IgG class switch DNA recombination by activating human B cells through an innate pathway that requires TLR9 and cooperates with IL-10," Journal of Immunology, vol. 173, no. 7, pp. 44794491, 2004.

[46] M. Gururajan, J. Jacob, and B. Pulendran, "Toll-like receptor expression and responsiveness of distinct murine splenic and mucosal B-cell subsets," PLoS ONE, vol. 2, no. 9, article e863, 2007.

[47] M. Murakami, H. Yoshioka, T. Shirai, T. Tsubata, and T. Honjo, "Prevention of autoimmune symptoms in autoimmune-prone mice by elimination of B-1 cells," International Immunology, vol. 7, no. 5, pp. 877-882, 1995.

[48] E. Montecino-Rodriguez and K. Dorshkind, "New perspectives in B-1 B cell development and function," Trends in Immunology, vol. 27, no. 9, pp. 428-433, 2006.

[49] L. N. Ho, T. Uehara, C.-G. Chen, H. Kubagawa, and M. D. Cooper, "Constitutive tyrosine phosphorylation of the inhibitory paired Ig-like receptor PIR-B," Proceedings of the National Academy of Sciences of the United States of America, vol. 96, no. 26, pp. 15086-15090, 1999.

[50] A. Maeda, A. M. Scharenberg, S. Tsukada, J. B. Bolen, J.-P. Kinet, and T. Kurosaki, "Paired immunoglobulin-like receptor B (PIR-B) inhibits BCR-induced activation of Syk and Btk by SHP-1," Oncogene, vol. 18, no. 14, pp. 2291-2297, 1999.

[51] H. An, J. Hou, J. Zhou et al., "Phosphatase SHP-1 promotes TLR- and RIG-I-activated production of type I interferon by inhibiting the kinase IRAK1," Nature Immunology, vol. 9, no. 5, pp. 542-550, 2008.

[52] M. Gilliet, W. Cao, and Y.-J. Liu, "Plasmacytoid dendritic cells: sensing nucleic acids in viral infection and autoimmune diseases," Nature Reviews Immunology, vol. 8, no. 8, pp. 594606, 2008.

[53] S. L. Doyle, C. A. Jefferies, C. Feighery, and L. A. J. O’Neill, "Signaling by toll-like receptors 8 and 9 requires Bruton's tyrosine kinase," Journal of Biological Chemistry, vol. 282, no. 51, pp. 36953-36960, 2007.

[54] K.-G. Lee, S. Xu, E.-T. Wong, V. Tergaonkar, and K.-P. Lam, "Bruton's tyrosine kinase separately regulates NF $\kappa$ B p65RelA activation and cytokine interleukin (IL)-10/IL-12 production in TLR9-stimulated B cells," Journal of Biological Chemistry, vol. 283, no. 17, pp. 11189-11198, 2008.

[55] L. A. D. Bradley, A. K. Sweatman, R. C. Lovering et al., "Mutation detection in the X-linked agammaglobulinemia gene, BTK, using single strand conformation polymorphism analysis," Human Molecular Genetics, vol. 3, no. 1, pp. 79-83, 1994.

[56] S. Hashimoto, S. Tsukada, M. Matsushita et al., "Identification of Bruton's tyrosine kinase (Btk) gene mutations and characterization of the derived proteins in $35 \mathrm{X}$-linked agammaglobulinemia families: a nationwide study of Btk deficiency in Japan,” Blood, vol. 88, no. 2, pp. 561-573, 1996. 
[57] W. N. Khan, F. W. Alt, R. M. Gerstein et al., "Defective B cell development and function in Btk-deficient mice," Immunity, vol. 3, no. 3, pp. 283-299, 1995.

[58] C. V. Rothlin, S. Ghosh, E. I. Zuniga, M. B. A. Oldstone, and G. Lemke, "TAM Receptors Are Pleiotropic Inhibitors of the Innate Immune Response," Cell, vol. 131, no. 6, pp. 11241136, 2007.

[59] M. M. Whitmore, A. Iparraguirre, L. Kubelka, W. Weninger, T. Hai, and B. R. G. Williams, "Negative regulation of TLR-signaling pathways by activating transcription factor-3," Journal of Immunology, vol. 179, no. 6, pp. 3622-3630, 2007.

[60] H. J. Martin, M. L. Jae, D. Walls, and S. D. Hayward, "Manipulation of the toll-like receptor 7 signaling pathway by Epstein-Barr virus," Journal of Virology, vol. 81, no. 18, pp. 9748-9758, 2007.

[61] T. Uehara, M. Bléry, D.-W. Kang et al., "Inhibition of IgEmediated mast cell activation by the paired Ig-like receptor PIR-B," Journal of Clinical Investigation, vol. 108, no. 7, pp. 1041-1050, 2001.

[62] M. Bléry, H. Kubagawa, C.-C. Chen, F. Vély, M. D. Cooper, and E. Vivier, "The paired Ig-like receptor PIR-B is an inhibitory receptor that recruits the protein-tyrosine phosphatase SHP1," Proceedings of the National Academy of Sciences of the United States of America, vol. 95, no. 5, pp. 2446-2451, 1998.

[63] A. Maeda, M. Kurosaki, M. Ono, T. Takai, and T. Kurosaki, "Requirement of SH2-containing protein tyrosine phosphatases SHP-1 and SHP-2 for paired immunoglobulin-like receptor B (PIR-B)-mediated inhibitory signal," Journal of Experimental Medicine, vol. 187, no. 8, pp. 1355-1360, 1998.

[64] R. R. Hardy, K. Hayakawa, M. Shimizu, K. Yamasaki, and T. Kishimoto, "Rheumatoid factor secretion from human Leu1+ B cells," Science, vol. 236, no. 4797, pp. 81-83, 1987.

[65] P. Youinou, L. Mackenzie, P. Katsikis et al., "The relationship between CD5-expressing B lymphocytes and serologic abnormalities in rheumatoid arthritis patients and their relatives," Arthritis and Rheumatism, vol. 33, no. 3, pp. 339-348, 1990.

[66] J. A. Sowden, P. J. Roberts-Thomson, and H. Zola, "Evaluation of CD5-positive B cells in blood and synovial fluid of patients with rheumatic diseases," Rheumatology International, vol. 7, no. 6, pp. 255-259, 1987.

[67] E. A. Leadbetter, I. R. Rifkin, A. M. Hohlbaum, B. C. Beaudette, M. J. Shlomchik, and A. Marshak-Rothstein, "Chromatin-IgG complexes activate B cells by dual engagement of IgM and Toll-like receptors," Nature, vol. 416, no. 6881, pp. 603-607, 2002.

[68] S. Izui and R. A. Eisenberg, "Circulating anti DNArheumatoid factor complexes in MRL/1 mice," Clinical Immunology and Immunopathology, vol. 15, no. 3, pp. 536$551,1980$.

[69] Y. Gyotoku, M. Abdelmoula, and F. Spertini, "Cryoglobulinemia induced by monoclonal immunoglobulin $\mathrm{G}$ rheumatoid factors derived from autoimmune MRL/MpJ-lpr/lpr mice," Journal of Immunology, vol. 138, no. 11, pp. 3785-3792, 1987. 


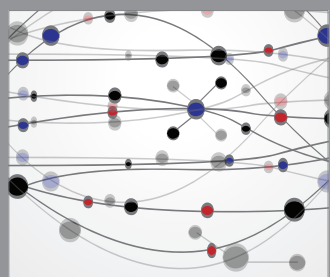

The Scientific World Journal
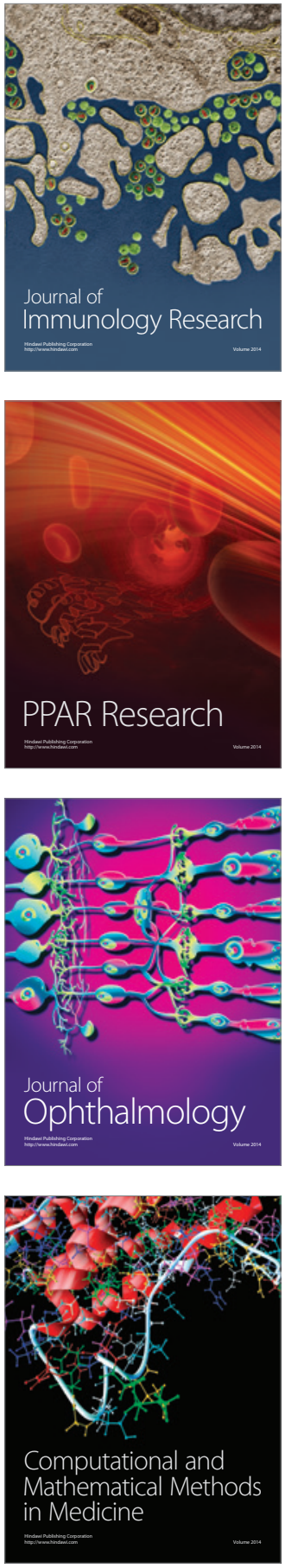

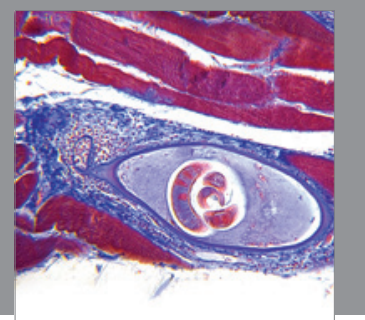

Gastroenterology

Research and Practice
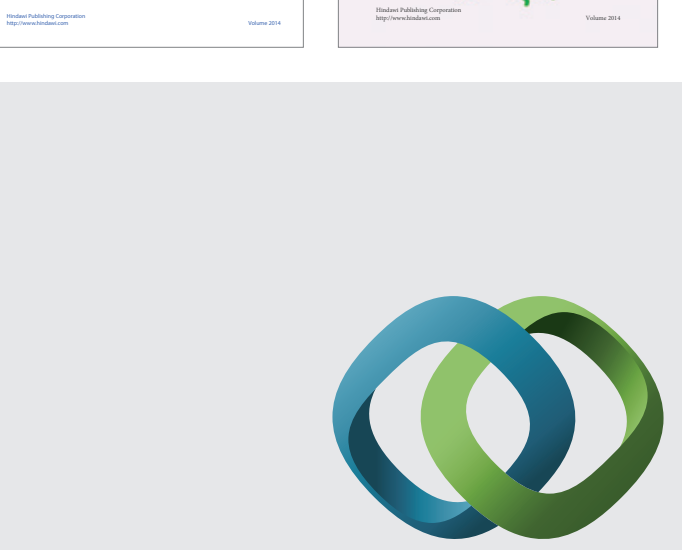

\section{Hindawi}

Submit your manuscripts at

http://www.hindawi.com
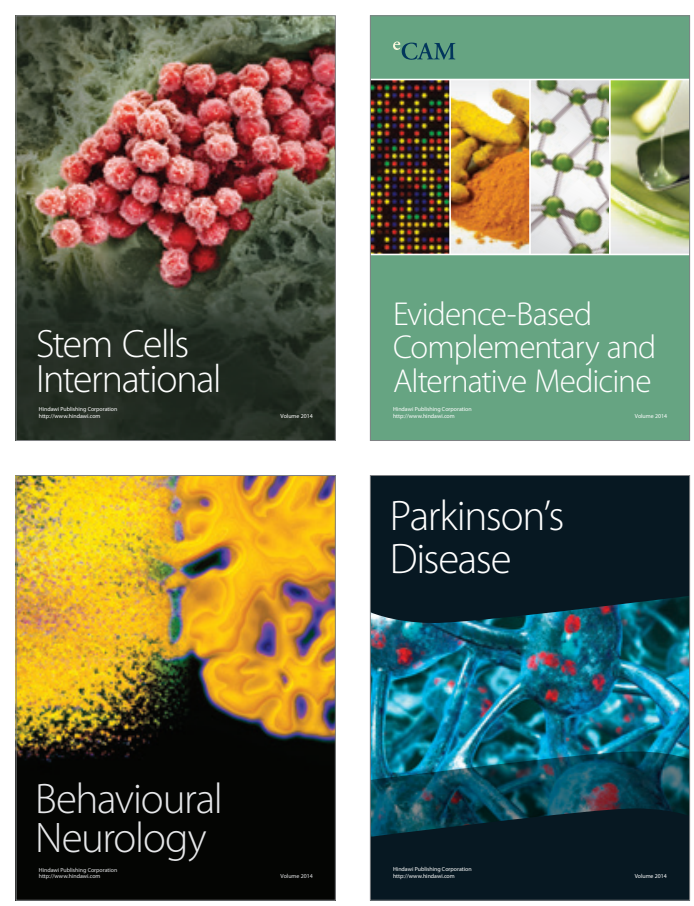

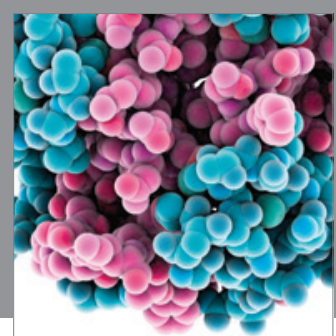

Journal of
Diabetes Research

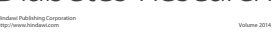

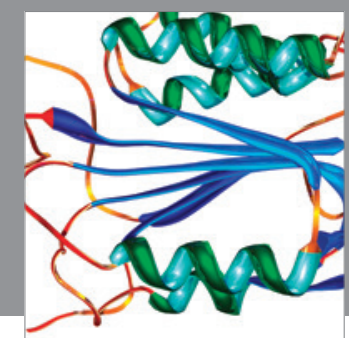

Disease Markers
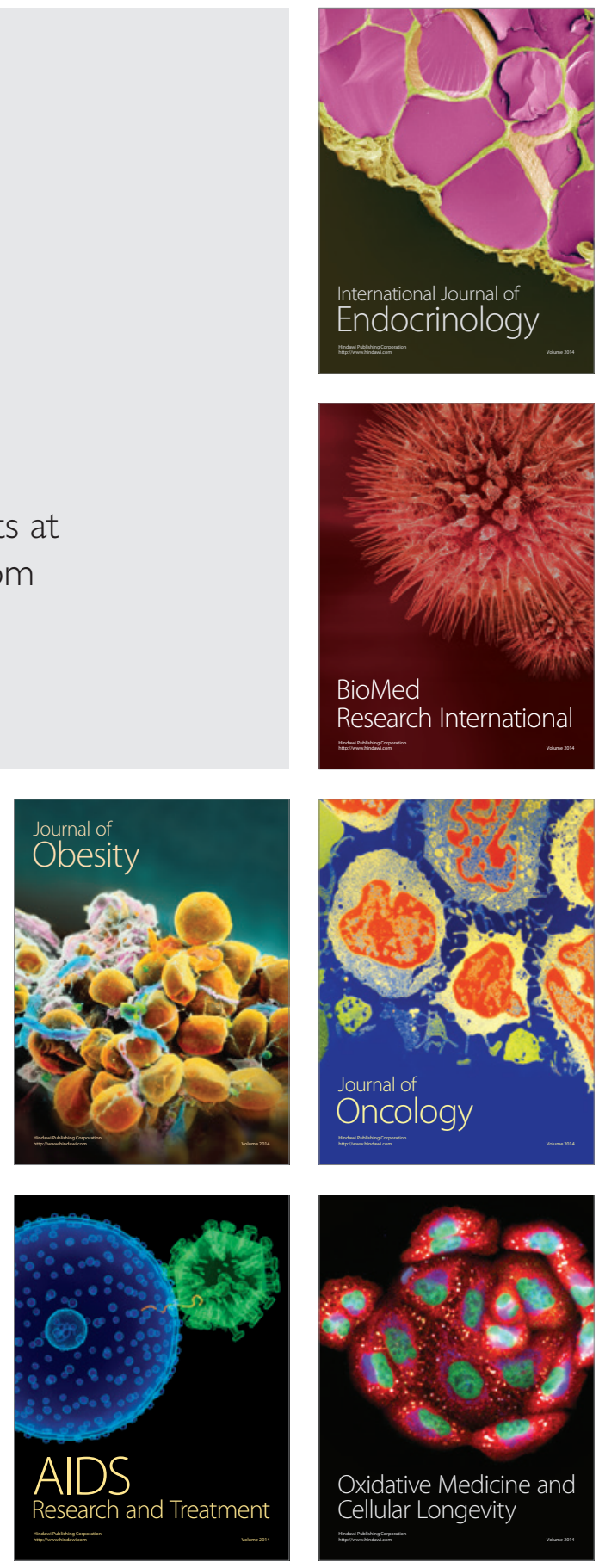